Dear Author,

Please, note that changes made to the HTML content will be added to the article before publication, but are not reflected in this PDF.

Note also that this file should not be used for submitting corrections. 


\title{
Q1 Transmission dynamics of HIV-1 subtype B in the Basque Country, Spain
}

\author{
Q2 J.A. Patiño-Galindo ${ }^{\mathrm{a}, \mathrm{b}}$, Michael M. Thomson ${ }^{\mathrm{c}}$, Lucía Pérez-Álvarez ${ }^{\mathrm{c}}$, Elena Delgado ${ }^{\mathrm{c}}$, María Teresa Cuevas ${ }^{\mathrm{c}}$, \\ Aurora Fernández-García ${ }^{\mathrm{b}, \mathrm{c}}$, Rafael Nájera ${ }^{\mathrm{c}}$, José A. Iribarren ${ }^{\mathrm{d}}$, Gustavo Cilla ${ }^{\mathrm{d}}$, Leyre López-Soria ${ }^{\mathrm{e}}$, \\ María J. Lezaun ${ }^{\mathrm{f}}$, Ramón Cisterna ${ }^{\mathrm{g}}$, F. González-Candelas ${ }^{\mathrm{a}, \mathrm{b}, *}$, \\ on behalf of Group of HIV-1 Antiretroviral Resistance Studies in the Basque Country \\ a Joint Research Unit "Infection and Public Health" FISABIO-Universitat de Valencia, Spain \\ b CIBER in Epidemiology and Public Health, Madrid, Spain \\ c Centro Nacional de Microbiología, Instituto de Salud Carlos III, Majadahonda, Madrid, Spain

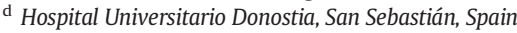 \\ e Hospital Universitario Cruces, Bilbao, Spain \\ ${ }^{\mathrm{f}}$ Hospital Universitario Araba, Vitoria, Spain \\ ${ }^{\mathrm{g}}$ Hospital Universitario Basurto, Bilbao, Spain
}

\section{A R T I C L E I N F O}

\section{Article history:}

Received 9 December 2015

Received in revised form 15 February 2016

Accepted 22 February 2016

Available online $\mathrm{xxxx}$

\section{Keywords:}

Transmission cluster

IDUs

MSM

Antiretroviral resistance

\begin{abstract}
A B S T R A C T
This work was aimed to study the HIV-1 subtype B epidemics in the Basque Country, Spain. 1727 HIV-1 subtype B 25 sequences comprising protease and reverse transcriptase (PR/RT) coding regions, sampled between 2001 and 26 2008, were analyzed. 156 transmission clusters were detected by means of phylogenetic analyses. Most of 27 them comprised less than 4 individuals and, in total, they included 441 patients. Six clusters comprised 10 or 28 more patients and were further analyzed in order to study their origin and diversification. Four clusters included 29 men who had unprotected homosexual sex (MSM), one group was formed by intravenous drug users (IDUs), and 30 another included both IDUs and people infected through unprotected heterosexual sex (HTs). Most of these 31 clusters originated from the mid-1980s to the mid-1990s. Only one cluster, formed by MSM, originated 32 after 2000. The time between infections was significantly lower in MSM groups than in those containing IDUs 33 (P-value $<0.0001$ ). Nucleoside RT and non-nucleoside RT inhibitor (NRTI and NNRTI)-resistance mutations to an- 34 tiretroviral treatment were found in these six clusters except the most recent MSM group, but only the IDU clus- 35 ters presented protease inhibitor (PI)-resistance mutations. The most prevalent mutations for each inhibitor class 36 were PI L90M, NRTI T215D/Y/F, and NNRTI K103N, which were also among the most prevalent resistant variants 37 in the whole dataset. In conclusion, while most infections occur as isolated introductions into the population, the 38 number of infections found to be epidemiologically related within the Basque Country is significant. Public health 39 control measures should be reinforced to prevent the further expansion of transmission clusters and resistant 40 mutations occurring within them.
\end{abstract}

(C) 2015 Published by Elsevier B.V. 42

\section{Introduction}

Since the detection of the first cases of acquired immunodeficiency syndrome (AIDS) in the early 1980s, the pandemic caused by its main causal agent, the human immunodeficiency virus type 1 (HIV-1), has become one of the most important global health problems due to its mortality and morbidity. The latest UNAIDS/WHO report (2013) estimates a total of 35.3 (32.2-38.8) million people infected around the world. In 2012, there were 2.3 (1.9-2.7) million new HIV infections

\footnotetext{
* Corresponding author at: Joint Research Unit “Infection and Public Health" FISABIOUniversitat de Valencia, Instituto Cavanilles de Biodiversidad y Biología Evolutiva, Parc Científic de la Universitat de València, c/ Catedrático José Beltrán, 2, 46980 Paterna, Valencia, Spain.

E-mail address: fernando.gonzalez@uv.es (F. González-Candelas).
}

globally, which is a 33\% decrease with respect to 2001. In Western 60 European countries, such as Spain, subtype B is the most prevalent 61 among the 9 subtypes of HIV-1. The epidemic of this variant started 62 to spread rapidly among specific risk groups, such as men who have 63 sex with men (MSM) and intravenous drug users (IDUs). Although 64 the rate of infections in these groups decreased during the 1990s thanks 65 to the development of adequate prevention campaigns (UNAIDS/WHO, 66 2013; Zehender et al., 2010), later years have been characterized by a 67 continuous increment of sexually-related infections, mainly among 68 MSM, while parenteral infections have decreased (ECDC/WHO, 2010). 69

HIV-1 is a retrovirus of the genus Lentivirus. Retroviruses present 70 high evolutionary rates that usually lead to a high genetic diversity. 71 These features are caused by three main factors: polymerization errors 72 of the reverse transcriptase (RT) (Roberts et al., 1988), genetic recombi- 73 nation and an explosive proliferation that leads to enormous effective 74 
population sizes, which promote the action of natural selection, favoring those mutations that increase the biological fitness of the virus and eliminating the disadvantageous alleles (Moya et al., 2004). These factors have important clinical consequences, such as the rise and spread of mutations related to resistance to antiretroviral drugs, but they also allow the reconstruction of the epidemic history of the virus by using phylogenetic tools (Holmes, 2004).

In the field of molecular epidemiology it is considered that epidemiologically related sequences should group together in a phylogenetic tree, forming transmission clusters, because they all share a common, recent ancestor (Hue et al., 2004, 2005). Coalescent methods for estimating phylogenetic trees (Kingman, 1982; Donnelly and Tavaré, 1995) are used to associate the divergence times from the common ancestor of the sampled individuals in a population with their demographic history. Thus, the results obtained offer information about dates of the introduction of viral variants in populations, the growth rate of infections during the epidemic and the most vulnerable groups of people to the virus (Moya et al., 2004).

The efficacy of highly active antiretroviral therapy (HAART) introduced in the 1990s is hampered by the emergence of resistance mutations in HIV-1 (Costagliola et al., 2007). Genotypic tests of resistance to antiretroviral drugs, after sequencing of the protease and reverse transcriptase (PR/RT) regions, are carried out routinely in many countries, including Spain, both for the design of individualized antiretroviral treatments and for the assessment of the frequency of certain resistances in the population (Costagliola et al., 2007). The widespread use of these tests has led to large, publicly accessible data sets of HIV-1 sequences that, combining analyses of their evolutionary history with epidemiological data, allow depicting the epidemic as well as characterizing its phylodynamics (Hue et al., 2004; Bello et al., 2010; Kouyos et al., 2010).

Genotypic tests of resistance for samples from the Basque country have been performed since 2001. The epidemic in the Basque Country has previously been analyzed using molecular data by Cuevas et al. (2009), who reported the existence of five major HIV-1 subtype B transmission clusters, with sizes ranging between 7 and 18 patients. Here, we have used a larger number of samples and we have also included a representative set of reference sequences to perform a more detailed phylogenetic analysis.

The objective of the present study was to characterize the epidemic of HIV- 1 subtype B in this region and analyze the transmission dynamics of some relevant cases. For this, we have used a dataset of 1727 sequences comprising HIV-1 PR/RT genomic regions obtained from 2001 to 2008 in the Basque Country as part of the genotyping program for the search of drug resistance mutations. The largest HIV-1 subtype $B$ transmission clusters detected were subjected to dated phylogenetic analysis (Drummond and Rambaut, 2007). The prevalence of mutations associated with antiretroviral drug resistance was estimated. The results obtained may help in the design of proper HIV prevention campaigns and treatments in this region.

\section{Methods}

\subsection{Dataset}

A total of 2497 HIV sequences $1200 \mathrm{nt}$ long and spanning the full PR and partial RT coding regions were obtained from patients attending the main health centers in the Basque Country, Spain (cities of Bilbao, San Sebastián and Vitoria) from 2001 to 2008. In cases of multiple sequences from a single patient, only the earliest one was included. Thus, each viral sequence represented a different patient. Additionally, 8504 worldwide sequences were retrieved from Los Alamos HIV dataset (http://www.hiv.lanl.gov) and were used as reference sequences to ensure the validity of the transmission chains detected in the Basque sample. All the sequences were aligned with MUSCLE v3.5 (Edgar, 2004).

\subsection{Phylogenetic reconstruction}

In order to identify transmission clusters, defined as viral lineages 139 derived from the same variant in the Basque population, two phyloge- 140 netic trees for the dataset of sequences which included both the Basque 141 and the reference sequences (11,001 sequences in total) were obtained 142 using FastTree 2.1 software (Price et al., 2010)) using the GTR + Г (4 143 categories) substitution model: (i) a tree obtained from a full codon 144 alignment, in which 40 codons associated with major resistance in PR 145 $(30,32,46,47,48,50,54,58,74,76,82,83,84,88,90)$ and RT $(41,62,146$ $65,67,69,70,74,75,77,100,101,103,106,108,115,116,151,181,147$ $184,188,190,210,215,219$ y 225) (Johnson et al., 2013) were removed, 148 yielding a total length of $1080 \mathrm{nt}$, and (ii) a tree obtained from only 149 third-codon positions of the original alignment (length of sequences 150 $=400 \mathrm{nt}$ ).

We considered as potential transmission clusters those clades 152 formed by at least 2 sequences of Basque origin present in both trees 153 with SH-like local support $\geq 0.90$ (Christin et al., 2012). Furthermore, 154 clusters with support between 0.90 and 0.95 , and/or including at least 155 10 patients, were further validated after their joint analysis with three 156 random datasets of 1000 subtype B reference sequences (full codon 157 alignments without resistance mutations). Basque sequences included 158 in these clusters were incorporated to the three datasets and analyzed 159 by maximum-likelihood with PhyML 3.0 (Guindon et al., 2010). Clusters 160 with Basque Country-only sequences were considered only if they had 161 Chi2-based approximate Likelihood-ratio test (aLRT) support $>0.999$. 162 Clusters were classified depending on the major transmission route 163 (>50\%) for the corresponding patients.

Only HIV-1 subtype B clusters were considered for further analysis. 165 All the sequences were subtyped with the REGA HIV-1 Subtyping Tool 166 - Version 2.0 (http://dbpartners.stanford.edu/RegaSubtyping/; De 167 Oliveira et al., 2005).

\subsection{Dated phylogenies}

The molecular clock signal of each transmission group equal to or 170 larger than 10 individuals was assessed by performing linear regression 171 analyses between the parameters "root-to-tip divergence" and 172 "sampling date" with the software Path-O-Gen v1.4 (Drummond et al., 173 2003), using the phylogenetic trees from each transmission cluster, 174 obtained as subtrees from the full-codon FastTree tree, as input. 175

These transmission groups were further analyzed using the full 176 codon alignments of $1080 \mathrm{nt}$. Dated phylogenies were obtained using 177 a Bayesian MCMC coalescent method, as implemented in BEAST v1.8.1 178 (http://beast.bio.ed.ac.uk/; Drummond and Rambaut, 2007). The 179 SRD06 model, which partitions by codon position $\left(\mathrm{HKY}_{112}+\Gamma_{112}\right), 180$ was used for all the BEAST analyses, as it fits better in most viral 181 protein-coding regions (Shapiro et al., 2006). A log-normal prior (medi- 182 an $=0.002$ substitutions per site and year, s/s/y, 95\% HPD upper limit $=183$ $0.0039 \mathrm{~s} / \mathrm{s} / \mathrm{y}$ ) was placed on the ucld.mean parameter (Hue et al., 2005; 184 Zehender et al., 2010). Under a relaxed molecular clock model, the most 185 appropriate demographic model [either constant demographic size, 186 exponential growth, logistic growth or Bayesian Skyline Plot (BSP)] 187 was determined as the one with the lowest Akaike Information Criteri- 188 on (AIC) value (Baele et al., 2012).

For each transmission cluster we performed at least two indepen- 190 dent runs of Bayesian MCMC, with chain lengths ranging between 5191 and 10 million states, sampling every 10,000 generations. Subsequently, 192 these runs were combined after discarding a 10\% burn-in. All the param- 193 eters were estimated from an effective sampling size $>200$ using the 194 software Tracer 1.5 (http://tree.bio.ed.ac.uk/software/tracer/). Trees 195 generated from the two BEAST runs were combined and summarized 196 after discarding a $10 \%$ burn-in using TreeAnnotator (http://beast.bio. 197 ed.ac.uk/). 


\subsection{Detection of intra-subtype recombination}

Intra-subtype recombination might introduce spurious long branches in the phylogenies of the transmission clusters considered (Hughes et al., 2009). We grouped all sequences from these clusters and checked for the presence of recombination events by performing five different recombination analyses implemented in RDP3 software: RDP, Geneconv, Bootscan, Maxchi and Chimera (Martin et al., 2010; Martin and Rybicki, 2000; Padidam et al., 1999; Martin et al., 2005; Smith, 1992; Posada and Crandall, 2001). The criterion used to consider the existence of recombination was to obtain significant evidence of recombination in at least two different analyses.

\subsection{Estimates of time between infections in transmission clusters}

The internal branch lengths of the transmission clusters allow us to estimate the time between infections (Lewis et al., 2008). 95\% HDPs for the median time between infections at each transmission cluster were estimated from the tree files produced with BEAST. We obtained the median and the upper and lower 95\%HPD limits for the internal branch lengths of each tree (Lewis et al., 2008) using an in-house Perl script combined with an R-script (R Development Core Team, 2011). The distributions of the median internal branch lengths were compared among transmission groups by ANOVA tests. Tukey's tests were performed as post-hoc analyses.

\subsection{Estimate of prevalence of drug resistance mutations}

Mutations associated with resistance to PR and RT inhibitors (Johnson et al., 2013), both in the total dataset and in each of the transmission groups analyzed, were detected using the Stanford University HIV Drug Resistance Database [http://sierra2.stanford.edu/sierra/ servlet/JSierra, (Liu and Shafer, 2006)] and their prevalence was estimated. Only major mutations were taken into account for the protease gene.

\section{Results}

\subsection{Detection of transmission clusters}

Of the 2497 HIV-1 sequences analyzed, 2311 belonged to subtype B and had been obtained from a total of 1727 different patients. Of these, 833 corresponded to IDUs, 340 to people infected through unprotected heterosexual sex (HT), 181 to MSM, and 49 to people vertically infected (VERT). 175 sequences belonged to people infected through sexual contact, not specifying whether it was heterosexual or homosexual. No risk factor was known for 149 people.

A total of 156 HIV-1 subtype B transmission clusters were consistent with the two phylogenetic reconstructions obtained with FastTree (from full-codon and third-positions alignments). In total, 441 (25.5\%) sequences in the Basque country dataset were included in a transmission cluster. Most of these clusters (93.6\%) contained 4 individuals at most (Fig. 1A). Transmission clusters of IDUs were the most abundant (Fig. 1B), followed by groups formed by people infected through unprotected heterosexual sex (HT), MSMs, and clusters containing both IDUs and HTs (IDU/HT). IDU clusters encompassed the largest number of patients $(\mathrm{n}=126)$, followed by MSM $(\mathrm{n}=124)$, HT $(\mathrm{n}=75)$ and IDU/HT ( $\mathrm{n}=81$ ) (Fig. 1C). MSM were significantly more likely to group in a transmission cluster than patients from other risk groups (Fisher's exact test: P-value $=2.2 \mathrm{E}-4$; odds-ratio $=1.76,95 \%$ confidence interval $=1.30-2.37$ ). Only 6 of the detected clusters comprised at least 10 individuals that, altogether, represented $4.8 \%$ of the Basque sample ( 83 individuals). Four of these clusters were formed by MSM (clusters C, D, E and F), one was classified as an IDU cluster (cluster B) and another was classified as IDU/HT (cluster A). All of them were validated with the maximum likelihood analyses (all had aLRT $>0.99$ in the reconstructions with PhyML), and all but cluster B had been 257 reported previously by Cuevas et al. (2009): A (cluster M2 in Cuevas 258 et al.), C (M5), D (M1.3), E (M1.1, M1.2), F (M3). MSM were significantly 259 more likely to group in a large transmission cluster than UDIs and HTs 260 (Fisher's exact test: P-value $=2.00 \mathrm{E}-15$; odds-ratio $=8.95,95 \%$ confi- 261 dence interval $=5.12-15.84$ ).

\subsection{Bayesian coalescent analyses}

No evidence of intra-subtype recombination events was found in 264 any of the six transmission clusters considered. Fig. 2 shows the dated 265 phylogenies of the transmission clusters reconstructed with BEAST 266 using dated-tips and considering the demographic model that yielded 267 the lowest AIC value in each group. tMRCA estimates differed among 268 groups with the earliest date corresponding to group B (median 269 tMRCA $=1983.8$ ), and the latest to group D (2000.5) (Table 1). 270

The ANOVA test comparing the median lengths of internal branch 271 concluded that there were significant differences between transmission 272 clusters ( $\mathrm{F}=37,382.3, \mathrm{df}=5$ and 47,463.27, P-value $<2.2 \mathrm{E}-16$ ), being 273 significantly shorter in MSM than in IDU or IDU/HT transmission 274 clusters (all Tukey's test comparisons: $\mathrm{P}=0.00$; Table 1 ). The same 275 results (not shown) were obtained with estimates obtained using all 276 the sequences as contemporaneous.

\subsection{Resistance mutations in transmission clusters}

The prevalence of mutations associated with antiretroviral drug 279 resistance in each transmission cluster and in the complete dataset are 280 shown in Table 2. While mutations associated with resistance to prote- 281 ase inhibitors (PIs) were found only in one transmission group (B, most 282 prevalent mutation L90M: 0.30), all transmission groups except D 283 presented mutations associated with resistance to nucleoside and 284 non-nucleoside reverse transcriptase inhibitors (NRTIs and NNRTIs, 285 respectively). Groups A and B presented the largest number of NNRTI 286 and NRTI resistance mutations, respectively. Among NRTI mutations, 287 $\mathrm{T} 215 \mathrm{D} / \mathrm{Y} / \mathrm{F}$ had the highest prevalence (1.0 in cluster $\mathrm{F}, 0.50$ in cluster 288 B), followed by M184I/V ( 0.50 in cluster B, 0.27 in cluster A) and 289 M41L (0.40, also in group B). The prevalence of NNRTI-resistance 290 mutations was lower than those causing resistance to NRTI, with 291 $\mathrm{K} 103 \mathrm{~N}$ being the most prevalent one ( 0.36 and 0.20 in groups $\mathrm{A}$ and $\mathrm{B}, 292$ respectively), followed by G190A/S (0.20 and 0.19 in groups B and E, 293 respectively). In the complete subtype B Basque dataset, mutations as- 294 sociated with resistance to PIs were also present with low prevalence 295 except L90M and M46I/L (0.13 and 0.11, respectively). The most fre- 296 quent NRTI-resistance mutations were M184I/V (0.36), L215Y/F 297 (0.26), M41L (0.23), D67N (0.17), L210W (0.15), K70E/R (0.13), and 298 K219D/Q/E/R (0.12). The most frequent NNRT-resistance mutation 299 was K103N/S (0.22), the only one with prevalence $>0.10$.

\section{Discussion}

We have analyzed 1727 HIV-1 subtype B sequences from different 302 patients obtained from health centers in the Basque Country, Spain, 303 between 2001 and 2008 to assess the HIV-1 epidemics in this popula- 304 tion. The large size of the dataset and the time-span in which these 305 sequences were obtained provide enough confidence to consider the 306 results obtained in this work as representative of the epidemic scenario 307 of HIV-1 in this region.

The results obtained from this work suggest that the HIV-1 subtype 309 B epidemic in the Basque Country is characterized by a majority of infec- 310 tions occurring as isolated introductions of the virus, although a repre- 311 sentative $25.5 \%$ of the patients were included in transmission clusters, 312 which ranged in size between 2 and 18 individuals. This proportion is 313 much lower than the $47 \%$ sequences grouping in transmission clusters 314 found by Cuevas et al. (2009) among newly diagnosed individuals. 315 The smaller size of their dataset (261 vs 1727 patients), methodological 316 
A


differences in phylogenetic analyses, and the low number of subtype B reference sequences $(n=4)$ used in that study can explain the markedly different proportions of individuals included in transmission clusters between both analyses. An additional factor that might explain the differences observed is the inclusion of non-newly infected patients in our analyses, which may cluster with less frequency due to higher number of nucleotide substitutions (longer external branches).

We found 6 large clusters, with sizes ranging between 10 and 18 individuals, that represented almost $5 \%$ of the total Basque dataset. Five of these clusters had been reported previously by Cuevas et al. (2009). Cluster B was not detected previously, because none of its sequences was analyzed by these authors for the reasons explained above. Previous studies in different European populations found trans- 329 mission groups that were mainly formed by MSM (Hue et al., 2005; 330 Kouyos et al., 2010; Lewis et al., 2008). In fact, the four largest transmis- 331 sion clusters found in our analysis (C, D, E, F) were also formed by MSM. 332 Hence, although the MSM population was less frequent than other risk 333 groups in the Basque country sampling, they were the major group as- 334 sociated to transmission clusters. IDUs frequently clustered either as 335 the only risk factor or including also transmissions through unprotected 336 heterosexual sex (IDU/HT), HTs and MSM, thus portraying a more di- 337 verse scenario in which IDUs were present in most of the smaller trans- 338 mission groups. Such clustering of IDUs and HTs has seldom been 339 reported (Kouyos et al., 2010; Holmes et al., 1995). 
A



C
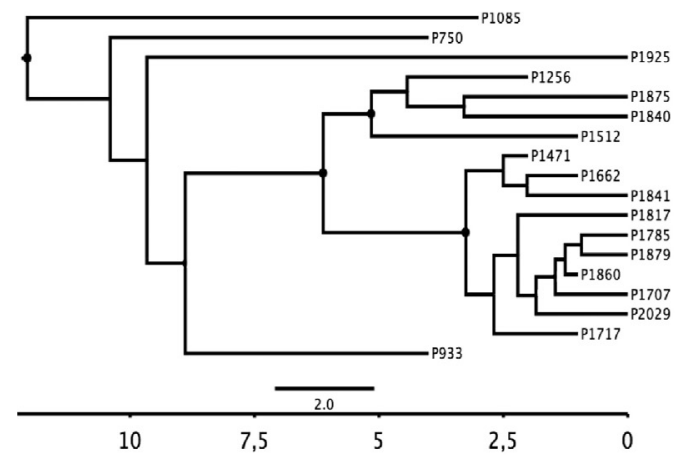

E
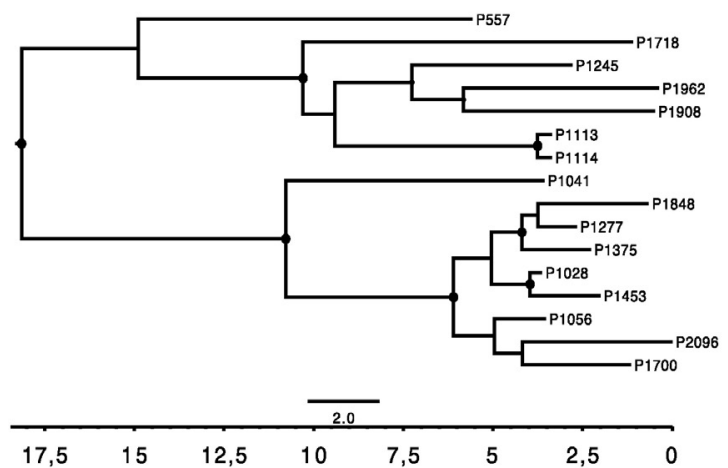

B

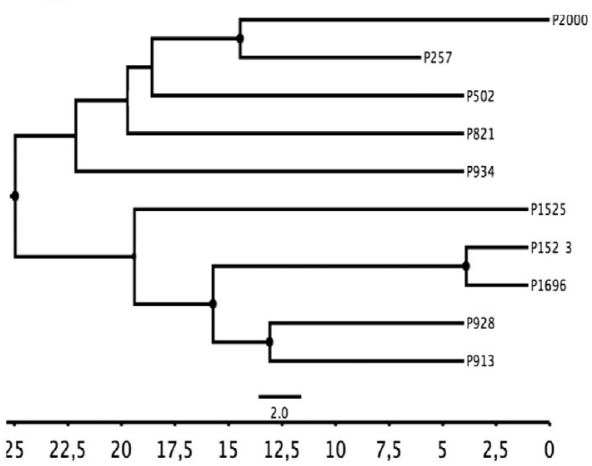

D
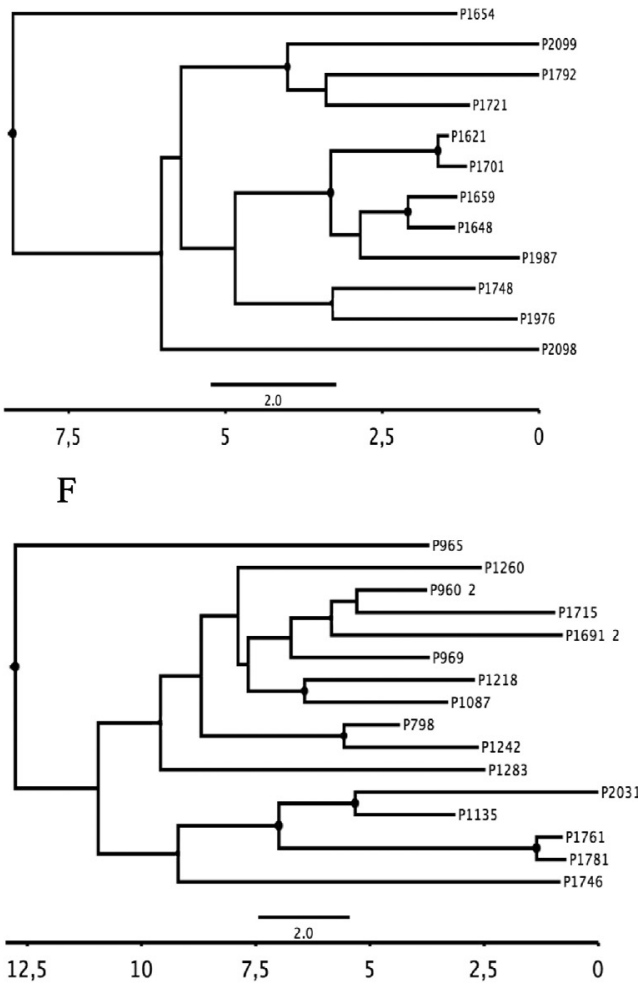


posterior probability $\geq 0.90$.

Previous studies in other European regions estimated that HIV-1 subtype B clusters initiated between the late 1960s and the early 1980s (Hue et al., 2005) or between the early 1990s and the beginning of 21st century (Lewis et al., 2008; Zehender et al., 2010).
Dated phylogenies showed the MRCAs from most clades to have diver- 345 sified from the mid-1980s to mid-1990s. The most recent clusters were 346 D and F, both formed by MSM. Their dates of origin (tMRCAs) are 347 coincident with the increase of infections among MSM after the 348

Table 1

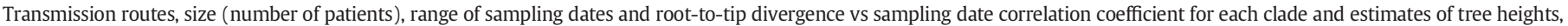
internal branch lengths and substitution rates as obtained with BEAST under the best fitting demographic model, using a relaxed molecular clock model, with tip dating.

\begin{tabular}{|c|c|c|c|c|c|c|c|c|c|}
\hline Cluster & Transmission & Taxa & Range* & Range (dates) & $\begin{array}{l}\mathrm{R} \text { (root-to-tip } \\
\text { divergence vs sampling } \\
\text { date correlation) }\end{array}$ & $\begin{array}{l}\text { Best fitting } \\
\text { demographic model }\end{array}$ & $\begin{array}{l}\text { Median tree height } \\
(95 \% \mathrm{HPD})^{*}\end{array}$ & $\begin{array}{l}\text { Median internal } \\
\text { branch lengths } \\
(95 \% \mathrm{HPD})^{*}\end{array}$ & $\begin{array}{l}\text { Median substitution } \\
\text { rate } x 10 e-3(95 \% \mathrm{HPD})^{\dagger}\end{array}$ \\
\hline A & IDU/HT & 11 & 5.57 & Nov 02-Jun. 08 & 0.03 & Exponential & 1986.3 (1980.6-1996.9) & $2.07(0.88-4.71)$ & $1.19(0.54-2.09)$ \\
\hline B & IDU & 10 & 4.30 & May 04-Oct. 08 & 0.46 & Logistic & $1983.8(1964.8-1996.1)$ & $3.08(1.36-7.18)$ & $1.54(0.67-2.73)$ \\
\hline C & Homosexual & 18 & 3.98 & Nov $04-$ Nov. 08 & 0.61 & Exponential & 1996.6 (1989.9-2001.6) & $0.61(0.30-1.31)$ & $2.20(1.17-3.38)$ \\
\hline $\mathrm{D}$ & Homosexual & 12 & 1.44 & Jun 07-Nov. 08 & $(-0.16)$ & Constant & 2000.5 (1992.8-2004.9) & $1.00(0.42-2.44)$ & $1.93(0.81-3.36)$ \\
\hline E & Homosexual & 16 & 5.57 & Apr 03-Nov. 08 & 0.25 & Logistic & $1990.8(1979.3-1998.9)$ & $1.39(0.61-3.09)$ & $1.66(0.80-2.73)$ \\
\hline $\mathrm{F}$ & Homosexual & 16 & 4.34 & Apr 04-Sept. 08 & 0.43 & Exponential & $1996.1(1988.1-2001.2)$ & $1.22(0.56-2.65)$ & $1.57(0.74-2.58)$ \\
\hline
\end{tabular}

* Time measured in years.

† Substitution per site and year. 
Table 2

Prevalence (proportion) of PI-NRTI- and NNRTI-resistance mutations in the HIV-1 subtype B Basque dataset ( $\mathrm{n}=1727$ sequences) and the six largest transmission clusters (A to F).



NRTI



NNRTI

\begin{tabular}{|c|c|c|c|c|c|c|c|c|c|c|c|c|c|c|}
\hline & V90I & A98G & L100I & K101 E/H/P & $\mathrm{K} 103 \mathrm{~N} / \mathrm{S}$ & V106 A/I/M & V108I & R138 A/G/K/Q/R & $\mathrm{V} 179 \mathrm{D} / \mathrm{E} / \mathrm{F} / \mathrm{T} / \mathrm{L}$ & $\mathrm{Y} 181 \mathrm{C} / \mathrm{I} / \mathrm{V}$ & $\mathrm{Y} 188 \mathrm{C} / \mathrm{L} / \mathrm{H}$ & G190A/S & $\mathrm{H} 221 \mathrm{Y}$ & $\mathrm{P} 225 \mathrm{H}$ \\
\hline Full dataset & 0.05 & 0.02 & 0.04 & 0.06 & 0.22 & 0.03 & 0.06 & 0.04 & 0.02 & 0.08 & 0.02 & 0.08 & 0.03 & 0.03 \\
\hline A & 0.09 & 0 & 0.09 & 0 & 0.36 & 0 & 0.09 & 0 & 0.09 & 0.09 & 0.09 & 0 & 0.09 & 0.09 \\
\hline B & 0 & 0 & 0 & 0.1 & 0.2 & 0 & 0 & 0 & 0.10 & 0.10 & 0 & 0.20 & 0 & 0.10 \\
\hline C & 0.06 & 0 & 0 & 0 & 0.06 & 0 & 0.06 & 0 & 0.06 & 0 & 0 & 0 & 0 & 0.06 \\
\hline D & 0 & 0 & 0 & 0 & 0 & 0 & 0 & 0 & 0 & 0 & 0 & 0 & 0 & 0 \\
\hline $\mathrm{E}$ & 0 & 0 & 0 & 0.13 & 0 & 0 & 0 & 0 & 0 & 0.13 & 0 & 0.19 & 0 & 0 \\
\hline $\mathrm{F}$ & 0 & 0 & 0 & 0 & 0 & 0 & 0 & 0 & 0 & 0.06 & 0 & 0.06 & 0 & 0 \\
\hline
\end{tabular}

commercialization of antiretroviral treatments and subsequent relaxation of prevention measures in this transmission group, especially a decrease of consistent condom usage (ECDC, 2013).

The estimated time between transmissions was significantly lower in MSM groups than in those including IDUs. Hughes et al. (2009) also found that HTs infected with HIV-1 subtypes A and C presented longer times between infections than MSM infected with HIV-1 subtype B. These results may be explained by the known high transmission risk of unprotected anal sex (Baggaley et al., 2010). In MSM who practice unprotected sex, the risk of HIV-1 infection is also increased due to role reversal during sexual intercourse: many individuals practice both insertive and receptive anal sex. This would increase HIV-1 spread by overcoming the low infection rates from receptive to insertive sexual partners (Beyrer et al., 2012).

NRTI resistance mutations were the most prevalent in the Basque dataset, with seven mutations present in more than $10 \%$ of the sampled sequences. PI and NNRTI resistance mutations were less frequent, with only two and one cases with a prevalence $>0.10$, respectively. For the six large transmission clusters, the prevalence of resistance mutations differed both among type of antiviral drug and among the analyzed transmission clusters. While only cluster B presented PI resistance mutations, all the clusters but one presented NRTI and NNRTI resistance mutations. It is important to mention the case of cluster F, in which all patients carry the low-level NRTI resistance mutation T215D. This possible example of drug resistance transmission in the Basque population has been reported previously (Cuevas et al., 2009; Vega et al., 2015). Hence, these results indicate that the dynamics of resistance mutations to antiretroviral drugs may differ among transmission clusters. However, this study lacks sufficient data to perform a detailed analysis of these patterns, and more extensive analyses are necessary to elucidate the factors originating these differences.

In conclusion, our results suggest an epidemic scenario of HIV-1 subtype $B$ in the Basque Country in which most infections appear to correspond to independent introductions in the population, although 383 there exist at least 6 major long-standing and diverse transmission 384 groups. Most of these groups are characterized by a large proportion 385 of MSM, in a disproportionally large frequency with respect to the pres- 386 ence of this risk group in the global sample. Furthermore, a shorter time 387 between infections among MSM relative to other risk groups demon- 388 strates the vulnerability of this collective to HIV-1 infections. Our results 389 reinforce the need to implement prevention campaigns in the MSM 390 population. This study also highlights the relevance and interest of ap- 391 plying Bayesian methods for phylogenetic and coalescent inference in 392 epidemiology.

\section{Acknowledgments}

We thank Dr. Daniel Zulaica, coordinator of the Plan for AIDS Preven- 395 tion and Control and Osakidetza-Basque Health Service for their support 396 in the development of the study.

397

The Spanish Group of HIV-1 Antiretroviral Resistance Studies in the 398 Basque Country: Álava-Hospital Txagorritxu: Agud J. M. Aldamiz M, 399 Ayensa C, Barroso J, Lezaun M. J, Michaus L, Pérez-Ortolá R, and Portu 400 J. J. H. de Santiago: Andia A and Labora A. Guipúzcoa-Complejo 401 Hospitalario Donostia: Arrizabalaga J, Cilla G, Echevarría J, Iribarren J. 402 A, Rodriguez-Arrondo F, Serrano-Bengoechea E, and Von Wichmann 403 M. A; H. de Zumarraga: Bustillo M.A. Vizcaya - H. de Galdákano: 404 López de Goicoechea M. J and Mayo J; H. de Cruces: Aguirrebengoa K, 405 López Soria L, Goikoetxea J, Montejo M, and Bereciartura E; H. de 406 Basurto: Baraia J. B, Cisterna R, Ezpeleta C, Ferrero O, Ibarra S, Imaz M, 407 Muñoz- Sánchez J, Santamaría J. M, Sota M, and Zubero Z; H. San Eloy: 408 Lizarraga M and Silvariño R.

This work was funded by Convenio de Colaboración entre 410 Osakidetza-Servicio Vasco de Salud y el Instituto de Salud Carlos III 411 (MVI 1158/03) and projects BFU2011-24112 and BFU2014-58656-R 412 from MINECO (Spanish Government). 


\section{References}

Baele, G., Lemey, P., Bedford, T., Rambaut, A., Suchard, M.A., Alekseyenko, A.V., 2012. Improving the accuracy of demographic and molecular clock model comparison while accommodating phylogenetic uncertainty. Mol. Biol. Evol. 29, 2157-2167.

Baggaley, R.F., White, R.G., Boily, M.C., 2010. HIV transmission risk through anal intercourse: systematic review, meta-analysis and implications for HIV prevention. Int. J. Epidemiol. 39, 1048-1063.

Bello, G., Aulicino, P., Ruchansky, D., Guimaraes, M., Lopez-Galindez, C., Casado, C., Chiparelli, H., Rocco, C., Mangano, A., Sen, L., Morgado, M., 2010. Phylodynamics of HIV-1 circulating recombinant forms 12_BF and 38_BF in Argentina and Uruguay. Retrovirology 7, 22.

Beyrer, C., Baral, S.D., van Griensven, F., Goodreau, S.M., Chariyalertsak, S., Wirtz, A.L., Brookmeyer, R., 2012. Global epidemiology of HIV infection in men who have sex with men. Lancet 380, 367-377.

Christin, P.A., Besnard, G., Edwards, E.J., Salamin, N., 2012. Effect of genetic convergence on phylogenetic inference. Mol. Phylogenet. Evol. 62, 921-927.

Costagliola, D., Descamps, D., Assoumou, L., Morand-Joubert, L., Marcelin, A.G., Brodard, V Delaugerre, C., Mackiewicz, V., Ruffault, A., Izopet, J., 2007. Prevalence of HIV-1 drug resistance in treated patients: a French nationwide study. J. Acquir. Immune Defic. Syndr. 46, 12-18.

Cuevas, M.T., Munoz-Nieto, M., Thomson, M.M., Delgado, E., Iribarren, J.A., Cilla, G., Fernandez-Garcia, A., Santamaria, J.M., Lezaun, M.J., Jimenez, L., Lopez-Soria, L.M. Sota, M., Contreras, G., Najera, R., Perez-Alvarez, L., 2009. HIV-1 transmission cluster with T215D revertant mutation among newly diagnosed patients from the Basque Country, Spain. J. Acquir. Immune Defic. Syndr. 51, 99-103.

De Oliveira, T., Deforche, K., Cassol, S., Salminen, M., Paraskevis, D., Seebregts, C., Snoeck, J., van Rensburg, E.J., Wensing, A.M.J., van de Vijver, D.A., Boucher, C.A., Camacho, R. Vandamme, A.M., 2005. An automated genotyping system for analysis of HIV-1 and other microbial sequences. Bioinformatics 21, 3797-3800.

Donnelly, P., Tavaré, S., 1995. Coalescents and genealogical structure under neutrality. Annu. Rev. Genet. 29, 401-421.

Drummond, A., Oliver, G., Rambaut, A., 2003. Inference of viral evolutionary rates from molecular sequences. Adv. Parasitol. 54, 331-358.

Drummond, A.J., Rambaut, A., 2007. BEAST: Bayesian evolutionary analysis by sampling trees. BMC Evol. Biol. 7, 214

ECDC, 2013. Men who have sex with men. Monitoring implementation of the Dublin Declaration on Partnership to Fight HIV/AIDS in Europe and Central Asia: 2012 progress report (www.ecdc.europa.eu ed).

ECDC/WHO, 2010. HIV/AIDS Surveillance in Europe 2009. European Centre for Disease Prevention and Control, Stockholm.

Edgar, R.C., 2004. MUSCLE: multiple sequence alignment with high accuracy and high throughput. Nucleic Acids Res. 32, 1792-1797.

Guindon, S., Dufayard, J.F., Lefort, V., Anisimova, M., Hordijk, W., Gascuel, O., 2010. New algorithms and methods to estimate maximum-likelihood phylogenies: assessing the performance of PhyML 3.0. Syst. Biol. 59, 307-321.

Holmes, E.C., 2004. The phylogeography of human viruses. Mol. Ecol. 13, 745-756.

Holmes, E.C., Zhang, L.Q., Robertson, P., Cleland, A., Harvey, E., Simmonds, P., Leigh Brown, A.J., 1995. The molecular epidemiology of human immunodeficiency virus type 1 in Edinburgh. J. Infect. Dis. 171, 45-53.

Hue, S., Clewley, J.P., Cane, P.A., Pillay, D., 2004. HIV-1 pol gene variation is sufficient for reconstruction of transmissions in the era of antiretroviral therapy. AIDS 18, 719-728.

Hue, S., Pillay, D., Clewley, J.P., Pybus, O.G., 2005. Genetic analysis reveals the complex structure of HIV-1 transmission within defined risk groups. Proc. Natl. Acad. Sci. U. S. A. $102,4425-4429$.
Hughes, G.J., Fearnhill, E., Dunn, D., Lycett, S.J., Rambaut, A., Leigh Brown, A.J., on behalf of 468 the UK HIV Drug Resistance Collaboration, 2009. Molecular phylodynamics of the 469 heterosexual HIV epidemic in the United Kingdom. PLoS Pathog. 5, e1000590. 470

Johnson, V.A., Calvez, V., Gunthard, H.F., Paredes, R., Pillay, D., Shafer, R.W., Wensing, A.M., 471 Richman, D.D. 2013. Update of the drug resistance mutations in HIV-1: March 2013. 472 Top. Antivir. Med. 21, 6-14.

Kingman, J.F.C., 1982. The coalescent. Stoch. Process. Appl. 13, 235-248. Weber, R., Hirschel, B., Cavassini, M., Furrer, H., Battegay, M., Vernazza, P.L., 476 Bernasconi, E., Rickenbach, M., Ledergerber, B., Bonhoeffer, S., Günthard, H.F., 2010. 477 Molecular epidemiology reveals long-term changes in HIV type 1 subtype B transmis- 478 sion in Switzerland. J. Infect. Dis. 201, 1488-1497.

Lewis, F., Hughes, G.J., Rambaut, A., Pozniak, A., Leigh Brown, A.J., 2008. Episodic sexual 480 transmission of HIV revealed by molecular phylodynamics. PLoS Med. 5, e50. 481

Liu, T.F., Shafer, R.W., 2006. Web resources for HIV type 1 genotypic-resistance test 482 interpretation. Clin. Infect. Dis. 42, 1608-1618.

Martin, D., Rybicki, E., 2000. RDP: detection of recombination amongst aligned sequences. 484 Bioinformatics 16, 562-563.

Martin, D.P., Lemey, P., Lott, M., Moulton, V., Posada, D., Lefeuvre, P., 2010. RDP3: a flexible 486 and fast computer program for analyzing recombination. Bioinformatics 26, 487 2462-2463.

Martin, D.P., Posada, D., Crandall, K.A., Williamson, C., 2005. A modified bootscan 489 algorithm for automated identification of recombinant sequences and recombination 490 breakpoints. AIDS Res. Hum. Retrovir. 21, 98-102.

Moya, A., Holmes, E.C., González-Candelas, F. 2004. The population genetics and 492 evolutionary epidemiology of RNA viruses. Nat. Rev. Microbiol. 2, 279-288. 493

Padidam, M., Sawyer, S., Fauquet, C.M., 1999. Possible emergence of new geminiviruses by 494 frequent recombination. Virology 265, 218-225.

Posada, D. Crandall, KA, 2001. Evaluation of methods for detecting recombination from 496 DNA sequences: computer simulations. Proc. Natl. Acad. Sci. U. S. A. 98, 13757-13762. 497

Price, M.N., Dehal, P.S., Arkin, A.P., 2010. FastTree 2 - approximately maximum-likelihood 498 trees for large alignments. PLoS One 5, e9490. ing. (http://www.R-project.org/ed. Vienna, Austria). 501

Roberts, J.D., Bebenek, K., Kunkel, T.A., 1988. The accuracy of reverse transcriptase from 502 HIV-1. Science 242, 1171-1173. 503

Shapiro, B., Rambaut, A., Drummond, A.J., 2006. Choosing appropriate substitution models 504 for the phylogenetic analysis of protein-coding sequences. Mol. Biol. Evol. 23, 7-9. 505 Smith, J.M., 1992. Analyzing the mosaic structure of genes. J. Mol. Evol. 34, 126-129. 506 UNAIDS/WHO, 2013. Global Report: UNAIDS Report on the Global AIDS Epidemic 507 (Geneva).

Vega, Y., Delgado, E., Fernández-García, A., Cuevas, M.T., Thomson, M.M., Montero, V., 509 Sánchez, M., Sánchez, A.M., Pérez-Álvarez, L., Spanish Group for the Study of New 510 HIV, 2015. Epidemiological surveillance of HIV-1 transmitted drug resistance in 511 Spain in 2004-2012: relevance of transmission clusters in the propagation of 512 resistance mutations. PLoS One 10, e0125699. 513

Zehender, G., Ebranati, E., Lai, A., Santoro, M.M., Alteri, C., Giuliani, M., Palamara, G., Perno, 514 C.F., Galli, M., Lo, P.A., Ciccozzi, M., 2010. Population dynamics of HIV-1 subtype B in a 515 cohort of men-having-sex-with-men in Rome, Italy. J. Acquir. Immune Defic. Syndr. 516 $55,156-160$. 